\title{
AVRs and PSSs Revisited
}

\author{
Guillermo Castro ${ }^{1}$, Juan Bermúdez ${ }^{2}$, Manuel Jiménez ${ }^{2}$, Manuel Alvarez ${ }^{3}$, Alex Arreaza ${ }^{4}$ \\ ${ }^{1}$ Inelectra, Caracas, Venezuela \\ guillermojose.castro@inelectra.com \\ ${ }^{2}$ Simon Bolívar University, Caracas, Venezuela \\ jbermude@usb.ve,09-10426@usb.ve \\ ${ }^{3}$ Lulea University of Technology, Lulea, Sweden \\ manuel.alvarez@ltu.se \\ ${ }^{4}$ Derwick Associates of Venezuela, C.A, Caracas, Venezuela \\ arreaza@derwick.com
}

\begin{abstract}
In this paper a conceptual analysis on the effect of Automatic Voltage Regulators (AVR) and Power System Stabilizers (PSS) for small signal stability (SSS) of power systems is developed. A modal formulation approach including the use of the Participation Matrix is used. The Modal Formulation is performed considering synchronous generator (SG) equations for both the Electromotive Forces (EMF) model and the Flux Linkages (FL) model as well as AVR and PSS equations. Then, a selected study case is evaluated to study the effect of the AVR and PSS on the small signal stability. For pedagogical purposes a comparison between the basic RLC circuit and a synchronous generator connected to an infinite bus, to clarify the concepts of synchronizing torque and damping torque, is presented.
\end{abstract}

\section{Introduction}

This work has been driven by the fact that the effect of Automatic Voltage Regulators (AVR) and Power System Stabilizers (PSS) on small signal stability (SSS) of power systems is not fully detailed in the literature. Key concepts in this subject remain relatively diffuse. For instance, some authors consider Electromotive Forces (EMF) and other ones consider Flux Linkages (FL) as state variables to model synchronous generators. In this paper a comparison between both models is made, in order to evaluate their effect on SSS of power systems, and for showing the principal process of linearization to derive the state space model needed in the modal formulation for a multi machine power system. In order to provide deep-in comprehension on the concepts of synchronous torque and damping torque, which are the principal components to evaluate the effect of AVR and PSS, a comparison between basic RLC circuit model and a synchronous generator (SG) connected to an infinite bus is elaborated.

\section{Modal Formulation and Participation Matrix}

For evaluating the effect of AVR and PSS on SSS the modal formulation is proposed. Consequently, the state space model of a multi machine system must be found. It is necessary to calculate the eigenvalues of the state Jacobian matrix $\underline{A}$ (see equation 1), which contains the "genetic" information of the system. If all eigenvalues have negative real part, the system is stable; on the contrary, if at least one eigenvalue has positive real part, the system becomes unstable.

In the latter case, the instability of the system is caused by the AVR just if the eigenvalue is an electromechanical (rotor) mode, which is a conjugate complex eigenvalue with the highest participation over the linearized torque angle $\left(\Delta \delta_{k}\right)$ measured w.r.t. the slack bus. For the $n$ machines case, there will be $n-1$ electromechanical modes. To determine the electromechanical modes, the Participation Matrix is used [5].

An electromechanical mode with positive real part, regarding the AVR is an indication of a negative damping torque introduced by the AVR itself. To solve this problem it is necessary introduce a positive damping component torque. This action is achieved by the PSS, traditionally.

\subsection{State Space Model}

The state space model is obtained considering the traditional linearized form, as follows:

$$
\begin{aligned}
& \underline{\Delta \dot{x}}=\underline{A} \underline{\Delta x}+\underline{B} \underline{\Delta u} \\
& \underline{\Delta y}=\underline{C} \underline{\Delta x}+\underline{D} \underline{\Delta u}
\end{aligned}
$$

\subsection{Participation Matrix}

In order to relate state variables with eigenvalues to identify electromechanical modes, the participation matrix is used considering the following form [2] [5]:

$$
\begin{gathered}
|\underline{P}|=\left|\underline{V} \bullet \underline{W^{t}}\right| \quad \text { (point to point product) } \\
\underline{W}=\underline{V}^{-1}
\end{gathered}
$$

Where $\underline{V}$ is the right-hand eigenvector matrix of Jacobian $\underline{A}$ and $\underline{W}$ is the left-hand eigenvector matrix of $\underline{A}$

\section{AVR and PSS Model}

The pedagogical AVR and PSS models used in this work are shown in Fig. 1. 


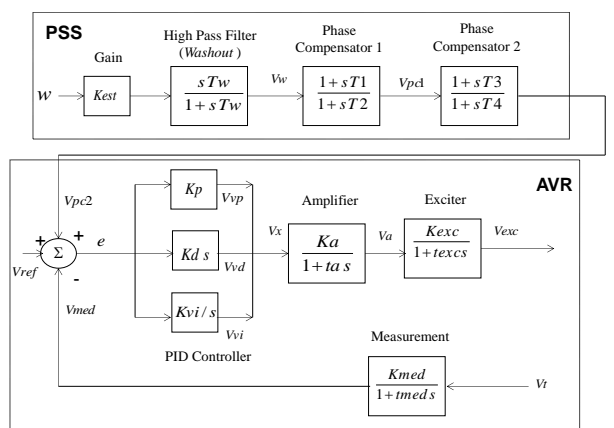

Fig. 1: AVR and PSS Model

The state variables Vmed, Vvi, Va, Vexc, Vw, Vpcl and $V p c 2$ are depicted in Fig. 1.

\section{EMF Model}

The synchronous generator equations related to the EMF model are as follows [3]:

$$
\begin{gathered}
\dot{w}=\frac{1}{2 H}\left(P m-P e-K_{d} w\right) \\
\dot{\delta}=w_{0} w \\
\dot{E q^{\prime}}=\frac{1}{t d o^{\prime}}\left(-E q^{\prime}+\left(X d-X d^{\prime}\right) I d+V e x c\right) \\
\dot{E d^{\prime}=}=\frac{1}{t q o^{\prime}}\left(-E d^{\prime}-\left(X q-X q^{\prime}\right) I q\right) \\
\dot{E q^{\prime \prime}}=\frac{1}{t d o^{\prime \prime}}\left(-E q^{\prime \prime}+\left(X d^{\prime}-X d^{\prime \prime}\right) I d+E q^{\prime}\right) \\
\dot{E d^{\prime \prime}}=\frac{1}{t q o^{\prime \prime}}\left(-E d^{\prime \prime}-\left(X q^{\prime}-X q^{\prime \prime}\right) I q+E d^{\prime}\right) \\
V q=-R a I q+X d^{\prime \prime} I d+E q^{\prime \prime} \\
V d=-R a I d-X q^{\prime \prime} I q+E d^{\prime \prime} \\
P e=E q^{\prime \prime} I q+E d^{\prime \prime} I d+\left(X d^{\prime \prime}-X q^{\prime \prime}\right) I q I d \\
P m=\text { constant }
\end{gathered}
$$

Where $w, \delta$ are rotor speed and angle deviations, $E$ stands for EMF along $\mathrm{d}$ and $\mathrm{q}$ axes, $V$ and $I$ are terminal voltage and current, $\mathrm{Pm}$ and $\mathrm{Pe}$ are shaft and air gap power, $H$ is the inertia constant and $w_{0}$ has been set to $2 \pi 60 \mathrm{rad} / \mathrm{s}$ in this study. All parameters are in per unit and correspond to those found in the equipment's data sheet.

Then, working in rectangular coordinates in both the network and the machine's rotor, the corresponding Jacobian matrix $\underline{A}$ is obtained [1]. Incremental and total saturation of synchronous generators can be included in this model considering the procedure proposed in [1].

\section{FL Model}

The synchronous generator equations related to Flux Linkages model are as follows [3]:

$$
\begin{aligned}
& \lambda q=L q^{\prime \prime} I q+K q 1 \lambda g+K q 2 \lambda k q \\
& \lambda d=L d^{\prime \prime} I d+K d 1 \lambda f+K d 2 \lambda k d
\end{aligned}
$$

$$
\begin{aligned}
& V q=-R a I q+w s \lambda d \\
& V d=- \text { RaId }-w s \lambda q \\
& P e=w s \lambda d I q-w s \lambda q I d \\
& \dot{\lambda f}=\frac{R f}{w s L d f} \operatorname{Vexc}+\frac{1}{D d}(-R f L k d \lambda f-R f L d f(L d f-L k d) I d+R f L d f \lambda k d) \\
& \dot{\lambda g}=\frac{1}{D q}(-\operatorname{Rg} L k q \lambda g-\operatorname{Rg} L q g(L q g-L k q) I q+R g L q g \lambda k q) \\
& \dot{\lambda k} d=\frac{1}{D d}(R k d L d f \lambda f-R k d L d f(L d f-L f f) I d-R k d L f f \lambda k d) \\
& \dot{\lambda k q}=\frac{1}{D q}(R k q L q g \lambda g-R k q L q g(L q g-L g g) I q-R k q L g g \lambda k q) \\
& D d=L k d L f f-L d f^{2} \\
& D q=L k q L g g-L q g^{2} \\
& K d 1=\frac{L d f(L k d-L d f)}{D d} \\
& K q 1=\frac{L q g(L k q-L q g)}{D q} \\
& K d 2=\frac{L d f(L f f-L d f)}{D d} \\
& K q 2=\frac{L q g(L g g-L q g)}{D q}
\end{aligned}
$$

Where, $\delta, w, \lambda f, \lambda g, \lambda k q, \lambda k d$ are state variables; $R a$ is stator resistance of phase "a" (positive sequence); $L d$ ", $L q$ " are subtransient non-saturated inductances on $\mathrm{d}$ and $\mathrm{q}$ axes respectively; $w s$ is the synchronous speed in per unit; $R f$ is the field winding resistance on d-axis; $R g$ is the hypothetic winding resistance on q-axis (for round rotors); $R k q$ is the damping winding resistance on q-axis; $R k d$ is the damping winding resistance on d-axis; $L d f$ is the non-saturated mutual inductance between stator winding and field winding on d-axis; Lqg is the non-saturated mutual inductance between the stator winding and the hypothetic winding "g" on q-axis (for round rotors); Lff is the non-saturated self-inductance of the field winding on d-axis; Lgg is the non-saturated self-inductance of the hypothetic "g" winding on q-axis (for round rotors); $\lambda d$ and $\lambda q$ are the flux linkages of the stator winding on $\mathrm{d}$-axis and q-axis respectively. The machine parameters in this model are not explicitly found in the equipment's data sheet and must be calculated [1]. The corresponding Jacobian matrix $\underline{A}$ is explicitly shown in [1]. Incremental and total saturation of synchronous generators can be included in this model by considering the procedure proposed in [1].

\section{Study Cases}

The test system used corresponds to the study case presented by Anderson \& Fouad in [4], consisting of 9 buses and 3 SG.

The system data considered in this evaluation was obtained from [1]. In order to evaluate the effect of AVR and PSS in the power system the following scenarios were studied:

- Case 1: G1, G2 and G3 in service with fast AVR and low load. 
- Case 2: G1, G2 and G3 in service with fast AVR and high load.

- Case 3: G1, G2 and G3 in service with fast AVR, low load and line 5-7 out of service.

- Case 4: G1, G2 and G3 in service with fast AVR, low load, line 5-7 out of service, PSS tuned in G2.

- Case 5: G1 and G3 in service. G2 is out of service. Fast AVR in G1 and G3.

For each case, only the electromechanical modes for each model (EMF and FL) are shown (which were obtained using the participation matrix).

\section{Case 1: G1, G2 and G3 in service with fast AVR and low load.}

The electromechanical modes obtained in this case are shown in Table 1.

Table 1. Electromechanical Modes Case 1

\begin{tabular}{|c|c|}
\hline EMF MODEL & FL MODEL \\
\hline$-0.6209 \pm 19.6492$ & $-0.8216 \pm 19.6118$ \\
\hline$-0.1804 \pm 12.7183$ & $-0.2720 \pm 12.6564$ \\
\hline
\end{tabular}

The results shown in Table 1 indicate that AVR does not introduce negative damping torque because all the electromechanical modes have negative real part. The results of both models are similar and have the same nature.

Case 2: G1, G2 and G3 in service with fast AVR and high load

The electromechanical modes obtained in this case are shown in Table 2.

Table 2. Electromechanical Modes Case 2

\begin{tabular}{|c|c|}
\hline EMF MODEL & FL MODEL \\
\hline $0.3833 \pm 11.8156$ & $0.1705 \pm 11.8853$ \\
\hline$-0.6587 \pm 19.5499$ & $-0.7857 \pm 19.5133$ \\
\hline
\end{tabular}

The results shown in Table 2 indicate that AVR introduce negative damping torque making the system unstable.

Case 3: G1, G2 and G3 in service with fast AVR, low load and line 5-7 out of service.

The electromechanical modes are shown in Table 3.

Table 3. Electromechanical Modes Case 3

\begin{tabular}{|c|c|}
\hline EMF MODEL & FL MODEL \\
\hline $0.1502 \pm 9.3880$ & $0.0651 \pm 9.3707$ \\
\hline$-0.6495 \pm 19.5470$ & $-0.8314 \pm 19.5109$ \\
\hline
\end{tabular}

The results shown in Table 3 indicate that AVR introduces negative damping torque, making the system unstable.

Case 4: G1, G2 and G3 in service with fast AVR, low load, line 5-7 out of service, PSS tuned in G2.
From Case 3, the power system is unstable because AVR introduces negative damping torque. Considering participation matrix, observing the $\Delta w_{2}$ row, it can be concluded that a PSS is required in G2. See Table 4.

Table 4. Electromechanical Modes Case 4

\begin{tabular}{|c|c|}
\hline EMF MODEL & FL MODEL \\
\hline$-5.6487 \pm 7.5353$ & $-4.3630 \pm 7.7190$ \\
\hline$-3.0144 \pm 16.8608$ & $-2.5665 \pm 17.6195$ \\
\hline
\end{tabular}

Now, the system becomes stable.

Case 5: G1 and G3 in service. G2 out of service. Fast AVR in G1 and G3. Line 5-7 out of service. Low load.

The electromechanical modes are shown in Table 5.

Table 5. Electromechanical Modes Case 5

\begin{tabular}{|c|c|}
\hline EMF MODEL & FL MODEL \\
\hline$-0.5701 \pm 15.9486$ & $-0.6532 \pm 15.7272$ \\
\hline
\end{tabular}

The results shown in Table 5 indicate that considering the same condition of Case 3 , but in a more severe scenario with G2 out of service, the power system is stable. These results indicate that AVR of G2 was the responsible to drive the system to instability. It is important to point out that if a PID controller is included in the AVR loop, the PSS might be required to introduce a phase lag, depending of the parameters of the AVR, instead of a phase lead, as is classically mentioned in the literature. Detailed results on this issue are shown in [1].

\section{RLC Circuit versus Synchronous Generator connected to an infinite bus}

In this section a pedagogical comparison between RLC series basic circuit and the classical problem of a SG connected to an infinite bus is presented in order to gain deep-in comprehension on the concepts of synchronous torque and damping torque. All quantities are in p.u. except the shaft speed ( $\mathrm{rad} / \mathrm{s})$.

Nomenclature of this section:

R, L, C: RLC series circuit parameters

$Q_{C}$ : Electric charge in the capacitor

$\mathrm{i}, \mathrm{v}$ : current and voltage.

$\delta$ : Torque angle (radians)

$\delta_{\mathrm{o}}$ : Initial torque angle.

$\omega$ : Rotor speed deviation ( $\omega$. .r.t nominal speed) .

$\omega_{0}$ : Nominal speed (i.e. $2 \pi 60 \mathrm{rad} / \mathrm{s}$ ).

$P_{m}$ : Mechanical (shaft) power.

$P_{e}$ : Electrical power.

$P_{d}$ : Damping power.

$K_{d}$ : Damping Power coefficient.

$K_{S}$ : Synchronizing coefficient.

$\Delta$ : Delta (differential increment) in any variable.

$\mathrm{H}$ : Machine inertia constant (MJoules/MVA) $\cong$ seconds.

$K_{m}=2 H / \omega_{0} \quad \zeta$ : Damping ratio

$P_{n}$ : Net accelerating power

$P_{n}=P_{m}-P_{e}-P_{d}$.

$P_{n}=P_{m}-K_{e} \cdot \sin (\delta)-K_{d} \cdot \omega$. 
$\Delta P_{n}=\Delta P_{m}-K_{s} . \Delta \delta-K_{d} \cdot \Delta \omega=\Delta P_{m}-\Delta P_{e}-\Delta P_{d}$

$K_{s}=K_{e} \cdot \cos \left(\delta_{o}\right)$.

$K_{e}=\left(E^{\prime} . V_{\infty}\right) / X$

$V_{\infty}=$ Infinite bus voltage in p.u. $=1\llcorner 0$.

$E^{\prime}=$ Transient internal EMF in p.u. $=\mathrm{E}\llcorner\delta$.

$\mathrm{X}=$ Total transient series reactance in p.u.

$=X_{\text {gen }}^{\prime}+X_{\text {stepup }}+X_{\text {line }}$

$\mathrm{s}=$ Generalized mode of oscillation or eigenvalue $=\sigma \pm \mathrm{j} . \omega$

\subsection{RLC series circuit versus SG in the context of SSS}

$$
V_{c}=\frac{1}{C} \cdot \int i(t) \cdot d t \Rightarrow V_{c}=\frac{1}{C} \cdot Q(t) \Rightarrow \Delta V_{c}=\frac{\Delta Q_{c}}{C}
$$

In the RLC series circuit expressed in p.u.:

$$
\begin{gathered}
V_{i n}=i . R+L . \frac{d i}{d t}+V_{C}=V_{R}+V_{L}+V_{c} \Rightarrow \\
\Delta V_{i n}=R . \Delta i+L \cdot \frac{d \Delta i}{d t}+\frac{\Delta Q_{C}}{C} \Rightarrow \\
\Delta V_{L}=\Delta V_{i n}-\Delta V_{C}-\Delta V_{R} ; \quad \zeta=\frac{0.5 R}{\sqrt{L / C}} ; W n=1 / \sqrt{L C} \\
L . \frac{d \Delta i}{d t}=\Delta V_{i n}-\frac{\Delta Q_{C}}{C}-R . \Delta i
\end{gathered}
$$

Equation (30) is identical in form to the swing equation of a SG operating against an infinite system, found in any power system text book [2].

$$
\begin{gathered}
K_{m} . \Delta \dot{\omega}=\Delta P_{m}-\Delta P_{e}-\Delta P_{d}=\Delta P_{n} \\
K_{m} . \Delta \dot{\omega}=\Delta P_{m}-K_{S} \Delta \delta-K_{d} \Delta \omega
\end{gathered}
$$

From equations (30) and (31) the following identification is possible (see Fig.2)

$$
\begin{aligned}
& \Delta V_{\text {in }} \Leftrightarrow \Delta P_{m} \quad \Delta Q_{C} \Leftrightarrow \Delta \delta \quad \Delta i \Leftrightarrow \Delta \omega \quad \Delta V_{C} \Leftrightarrow \Delta P_{e} \\
& \Delta V_{R} \Leftrightarrow K_{d} \Delta \omega \quad \Delta V_{L} \Leftrightarrow K_{m} . \Delta \dot{\omega}=\Delta P_{n} \quad \Delta \dot{\omega}=\frac{\Delta P_{n}}{K_{m}} \\
& R \Leftrightarrow K_{d} \quad L \Leftrightarrow \frac{2 . H}{\omega_{o}}=K_{m} \quad C \Leftrightarrow \frac{1}{K_{S}} \\
& \zeta=\frac{0.5 K_{d}}{\sqrt{K_{m} K_{s}}} \quad W n=\sqrt{K_{s} / K_{m}}
\end{aligned}
$$
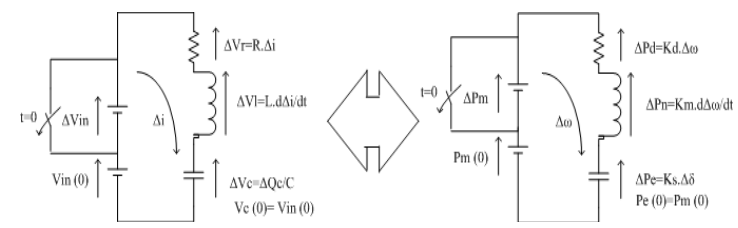

Fig. 2: Similarities between RLC circuit and SG

A plot of the electrical power and synchronizing coefficient versus $\delta$ is shown in Fig. 3.

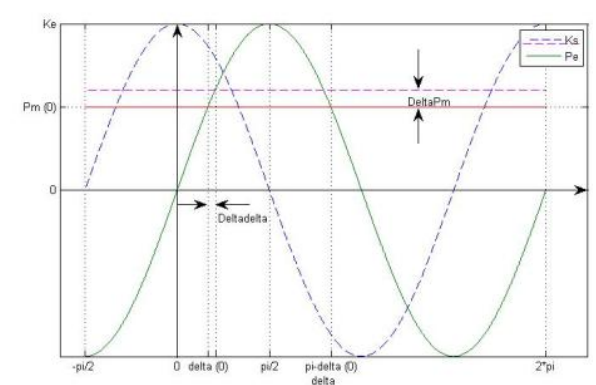

Fig. 3: Electric Power and Synchronizing Coefficient.

\subsection{The Behavior of the RLC Circuit}

If the driving (Input) d.c. voltage of an RLC series circuit is slightly increased, the elements "get in motion" such that the inductor stores magnetic energy, the capacitors stores electric energy and the resistor dissipates energy in order to damp the oscillations of energy established between the inductor and the capacitor. This oscillatory response goes on until the increment in $\Delta V_{i n}$ is reached by the capacitor voltage $\Delta V_{C}$, in whose case a new steady state is obtained and the current dies out in the circuit. In the meantime, energy is exchanged between the capacitor and the inductor: $\Delta \mathrm{E}_{\mathrm{L}}=0.5 * \mathrm{~L}^{*} \Delta \mathrm{V}_{\mathrm{L}}{ }^{2} ; \Delta \mathrm{Ec}=0.5 * \mathrm{C} * \Delta \mathrm{Vc}^{2}$

If the circuit were of order 1 (only one active element) such as an RL or RC circuit, the response could not be oscillatory, but exponential. The oscillations are damped out by the resistor. A null resistance causes sustained oscillations and a negative resistance would imply increasing oscillations.

\subsection{The Behavior of the Synchronous Generator}

A SG delivering power to an infinite system through a stepup transformer and a series transmission line behaves like the RLC series circuit when a $\Delta P_{m}$ step is applied in its shaft [6]. As time goes on, the torque angle rises implying an increment in rotor speed. Eventually the speed will regain its original value forced by the infinite bus, but in the meantime a damping torque acts due to mechanical friction and to the existence of rotating loads, in general. The original and final value of the rotor speed is zero, as it represents the deviations $\omega . r . t$ the nominal (initial) value.

From equations (30) and (31) and Fig. 2, many similarities between the RLC circuit and the SG can be drawn:

a) An increment in $P_{m}$ causes a natural increment in the rotor position $\delta$, as the rotor is "pushed forward" (compare with $V_{\text {in }}$ and $Q_{C}$ ).

b) Assuming that $\mathrm{Ks}>0(0 \leq \delta \leq \pi / 2)$ when the machine reaches its new steady state $\Delta \omega=0, \Delta P_{d}=0$, $\Delta P_{n}=0, \Delta P_{e}=\Delta P_{m}, \Delta \delta=\left(\Delta P_{m} / K_{S}\right)>0$. This case corresponds to the conventional behavior of the RLC circuits $\left(\Delta Q_{C}=C \cdot \Delta V_{\text {in }}\right.$ in steady state $)$

c) Assuming that $\mathrm{Ks}<0(\pi / 2 \leq \delta \leq \pi)$, under an increment in $\mathrm{Pm}$ the machine does undergo an increment in $\delta$, but a reduction in $\mathrm{Pe}$, since $\mathrm{Ks}$ is negative, implying that $\mathrm{Pn}$ cannot ever become zero, reason why the rotor speed would increase boundlessly. The machine protection relays would trip the machine out, no matter $\mathrm{Kd}$ is positive. This case corresponds to a negative $\mathrm{C}$ in the RLC circuit. Under such situation the generalized 
impedance of the capacitor $\mathrm{Zc}=[1 /(\mathrm{s} . \mathrm{C})]$ becomes negative, transforming itself into an inductor, speaking in general terms:

$$
\begin{aligned}
& Z_{L}=s \cdot L=[\sigma+j \cdot \omega] \cdot L \\
& Z_{C}=\frac{1}{s . C}=\frac{1}{[\sigma+j \cdot \omega]} \cdot \frac{1}{C}=\frac{\sigma-j \cdot \omega}{\sigma^{2}+\omega^{2}} \cdot \frac{1}{C}=\frac{\sigma-j \cdot \omega}{|s|^{2}} \cdot \frac{1}{C}
\end{aligned}
$$

When $\mathrm{C}$ becomes negative, the generalized impedance of the capacitor moves from the fourth to the second quadrant, coinciding with the nature of the quadrature part of $Z_{L}$. Accepting that under certain circumstances the capacitor "becomes an inductor", the RLC circuit cannot oscillate, since it becomes an RL circuit (order 1).

d) This kind of situation is found if the machine were operating at an initial point such as $\left(\pi-\delta_{o}\right)$, which happens to be an unstable equilibrium point, no matter $\mathrm{Kd}$ is positive

e) If $\mathrm{Kd}$ were negative (theoretically) the machine would become unstable even working in the nominal stable region $(0 \leq \delta \leq \pi / 2)$. That case corresponds to a negative value of $\mathrm{R}$ in the RLC circuit. Under certain conditions AVRs can introduce negative damping torques, which are dealt with PSS controllers as discussed previously.

f) Below $\delta=0$, Ks is positive because the corresponding reverse power "drives the machine" forward, towards the attraction point $\left(\delta_{o}\right)$. For $\delta>\pi \mathrm{Ks}$ is negative because the reverse power in that zone "drives the machine" away from its repulsion equilibrium point $\left(\pi-\delta_{o}\right)$, in either sense.

g) If the eigenvalues of the system (SG) were evaluated at $\delta=\delta_{o}$, they would result of complex conjugate nature with negative real part, for $\mathrm{Kd}>0$. When calculated at $\delta=\pi-\delta_{o}$, the eigenvalues would result nonoscillatory with positive real part, even for $\mathrm{Kd}>0$.

h) The Participation Matrix in absolute value would indicate that both $\Delta \delta$ and $\Delta \omega$ are affected by the "damaged" eigenvalue (complex conjugate), if evaluated at $\delta=\delta_{o}$. For $\delta=\pi-\delta_{o}$, the same analysis would indicate a non-oscillatory response, with the "damaged" eigenvalue (real value) affecting mainly the torque angle.

i) For $\delta=\pi / 2$ the machine has $\mathrm{Ks}=0$, which means $\mathrm{C}=\infty$ in the RLC case, implying that the generalized impedance of the capacitor becomes zero (short-circuit).

j) The energy balance as time goes on complies with:

$$
\begin{aligned}
& \Delta E_{\text {inductor }}=\frac{1}{2} \cdot L \cdot \Delta i^{2} \Leftrightarrow E_{\text {kinetic }}=\frac{1}{2} \cdot K_{m} \cdot \Delta \omega^{2} \\
& \Delta E_{\text {capacitor }}=\frac{1}{2} \cdot C \cdot \Delta V_{C}^{2} \Leftrightarrow E_{\text {potencial }}=\frac{1}{2} \cdot K_{S} \cdot \Delta \delta^{2} \\
& \Delta E_{\text {resistor }}=\int R \cdot \Delta i^{2} \cdot d t \Leftrightarrow E_{\text {damping }}=\int K_{d} \cdot \Delta \omega^{2} \cdot d t \\
& \Delta E_{\text {source }}=\int \Delta V_{\text {in }} \cdot \Delta i \cdot d t=\Delta E_{\text {inductor }}+\Delta E_{\text {capacitor }}+\Delta E_{\text {resistor }} \\
& \Delta E_{\text {source }} \Leftrightarrow \Delta E_{\text {mechanical }}=\int \Delta P_{m} \cdot \Delta \omega \cdot d t=\Delta E_{\text {kinetic }}+\Delta E_{\text {potencial }}+\Delta E_{\text {damping }}
\end{aligned}
$$

See Fig. 4 and 5 for a small applied $\Delta P_{m}$

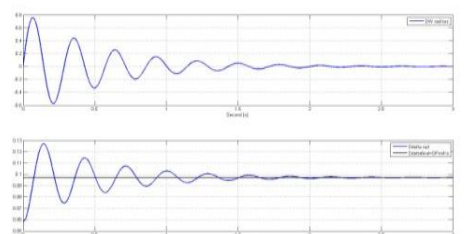

Fig. 4: $\Delta \omega$ and $\Delta \delta$ versus time under an increment $\Delta P_{m}$

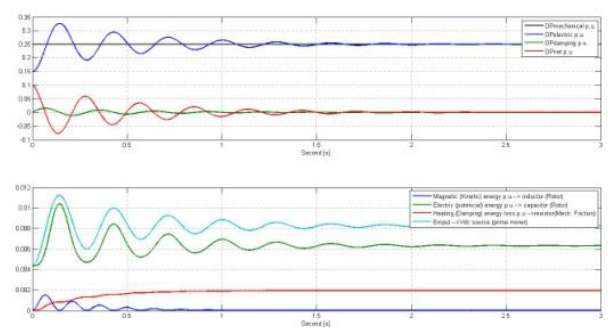

Fig. 5: Power and Energy versus time under an increment $\Delta P_{m}$

\section{Conclusions}

In this paper the EMF and FL model equations are clearly stated for the synchronous generator, in order to perform small signal analysis or even transient stability analysis. The results obtained using the two models are acceptable. The pedagogical comparison between RLC basic circuit and synchronous generator is a useful tool to understand the principle of synchronizing torque and damping torque, in order to comprehend the effect of AVR and PSS on small signal analysis of power system. The results obtained in the study cases indicate that when an electromechanical mode has positive real part, the power system is unstable due to a negative damping torque introduced by the AVR. The AVR causes the instability of the system especially in power system with high load levels, fast exciters or contingency (maintenance) conditions. Also, it is important to note that when the generator associated with the "damaged" electromechanical mode is out of service the related electromechanical mode disappears and the power system becomes stable. Finally, it is noted that PSS is required just when an electromechanical mode has positive real part and its task is to introduce positive damping torque to compensate the negative damping torque introduced by the AVR. When a PID controller exists in the AVR, it is possible that the PSS be required to introduce a phase lag instead of the classical phase lead action. These results are not included in this paper.

\section{References}

[1] G. J. Castro Veroes, "Conceptual Analysis of the Effect of AVR and PSS on Small Signal Analysis of Power System", M.S. thesis, CTE, USB, Caracas, 2014.

[2] P. Kundur, "Power System Stability and Control", McGraw-Hill California, USA, 1994.

[3] H. W. Dommel, "State of the Art of Transient Stability Simulation for Electric Power Systems", The University of British Columbia, California, 1977.

[4] P. M. Anderson, A. Fouad, "Power System Control and Stability", The Iowa State University Press, 1977.

[5] J. I. Pérez Arriaga, A. A Fouad, "Selective Modal Analysis with Application to Electric Power Systems", PhD. thesis, MIT, 1981

[6] J.F. Bermúdez, "Power Systems Analysis and Control", Technical Notes, USB, Caracas, 2015.

\section{Acknowledgements:}

The piece of research done by Professor J.F. Bermudez in this paper and his attendance to this Conference was sponsored by DERWICK ASSOCIATES OF VENEZUELA. 